\title{
Brightness contrast effects in a pupillometric experiment
}

\author{
ECKHARD H. HESS, PAUL W. BEAVER, and PATRICK E. SHROUT \\ University of Chicago, Chicago, Illinois 60637
}

\begin{abstract}
Pupillometric experiments which employ picture slides as stimuli have been criticized for their failure to control intrastimulus brightness variation. This factor has been suggested as the source of pupil size changes that are observed in response to picture slide stimuli, rather than the emotional response of the subject. The present study required subjects to direct their gaze to areas of a slide that varied in brightness. On the basis of the obtained results, it was concluded that brightness contrast does not play a major role in the pupil's response to picture slide stimuli which have been properly prepared.
\end{abstract}

Interest in the pupil as a psychophysical indication of affect has been generated by studies which report changes in pupil diameter in response to affective picture slide stimuli (cf. Hess, 1965). In these studies, the change in pupil diameter is measured from control shides of relatively uniform brightness to experimental slides of pictures. While the experimental slides are matched with the control slides in overall brightness, the experimental slides do have greater intrastimulus variability in the distribution of brightness. Lowenfeld (1966) suggests that such variation in brightness may account for the changes in pupil size attributed to the affective value of the picture. Recent reviews of pupillometrics echo this contention (Goldwater, 1972; Janisse, 1974).

Hess (1972) suggests that the influence of brightness contrast upon the pupil can be minimized through the employment of white and black Bourges sheet overlays. In addition, Hess goes on to recommend methods for measuring brightness contrast and criteria for acceptable levels of brightness contrast.

Little empirical evidence has thus far been offered which substantiates the argument that intrastimulus brightness contrast can account for the changes in pupil size in response to picture slide stimuli. In one study, Woodmansee (Note 1 and unpublished data) employed a white and black checkered square to measure the magnitude of pupil change to intrastimulus brightness variation. He found that the average percent change in pupil size from looking at light to dark squares to be only $2.1 \%$. In another study, Hess (1975, pp. 109-112) found the average change in pupil size produced by looking at white and black squares to be $2.5 \%$.

The current study was undertaken to determine the extent to which variation in the pupil response to picture slide stimuli can be attributed to pupil variance in response to intrastimulus brightness contrast when a maximum range of intrastimulus brightness values are employed-greater than the normal range characteristic of pictures. The procedures represent standard pupillometric methodology which has been developed and used in our laboratory (Hess, 1972).

\section{Subjects \\ METHOD \\ Thirty-two subjects, all students at the University of Chicago, were employed in this study.}

\section{Apparatus}

Pupil size was monitored by a TV pupillometer and camera, Model 1000. manufactured by the Space Sciences Division of the Whittaker Corporation, and was recorded by a Bausch and Lomb VOM 5 chart recorder, set at $10 \mathrm{mV}$. A slide was projected from behind a translucent screen. A Kodak Carousel slide projector was modified by inserting an opaque disk with a $6-\mathrm{mm}$ round hole in front of the lens to reduce image brightness. The screen was positioned $1 \mathrm{~m}$ from the subject. The projected slide measured $20 \mathrm{x}$ $12.5 \mathrm{~cm}$ on the screen, subtending a visual angle of $11.4 \mathrm{deg}$ on the horizontal plane and $6.9 \mathrm{deg}$ on the vertical plane. One achromatic experimental slide stimulus was employed. The slide consisted of four squares which varied in brightness from white to black on a constant medium gray background. The side of the squares measured $2.5 \mathrm{~cm}$ at the screen and subtended a visual angle of $1.5 \mathrm{deg}$ on both horizontal and vertical planes. When measured at the screen by a Gossen footcandle meter, the upper left square measured $18 \mathrm{fc}$, the upper right square measured $16 \mathrm{fc}$, the lower left square measured $9 \mathrm{fc}$, and the lower right square measured $6 \mathrm{fc}$, while the center and background of the slide measured $12 \mathrm{fc}$. A schematic representation of the slide is pictured in Figure 1.

\section{Procedure}

The subjects were each given time to adjust to and feel at ease with the experimental situation. A dry run was conducted to give the subjects practice with following the instructions. The experimenter instructed the subject when to look at the slide and which portion to view. Each portion of the slide was then viewed, at intervals of $8 \mathrm{sec}$. Half of the subjects were told to look at progressively brighter squares, and the other half were told to look at progressively darker squares.

\section{RESULTS}

The mean pupil sizes across all subjects for each brightness gradient were: $4.138 \mathrm{~mm}$ in response to the 


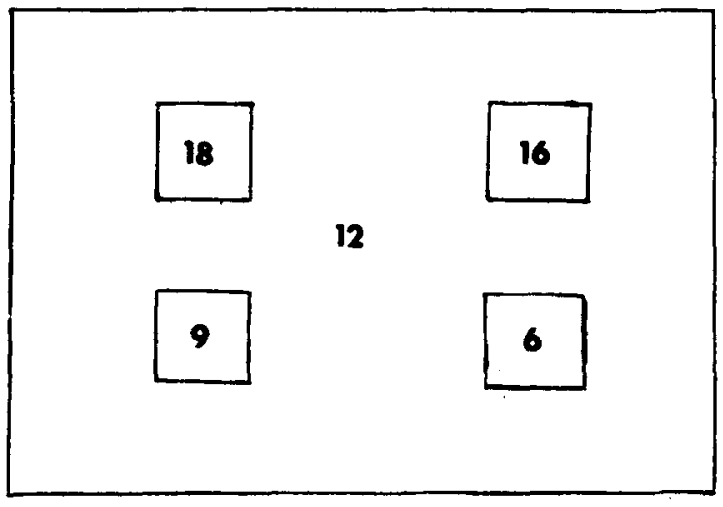

Figure 1. Schematic representation of experimental slide.

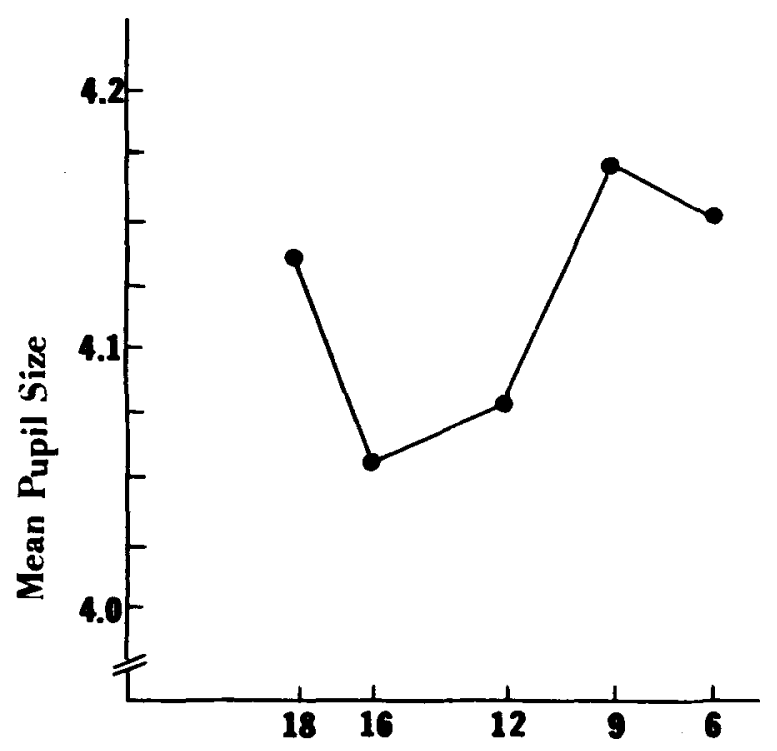

Brightness (fe)

Figure 2. Mean pupil size for each brightness category.

brightest area of $18 \mathrm{fc} ; 4.053 \mathrm{~mm}$ to $16 \mathrm{fc} ; 4.089 \mathrm{~mm}$ to $12 \mathrm{fc} ; 4.166 \mathrm{~mm}$ to $9 \mathrm{fc}$; and $4.147 \mathrm{~mm}$ to $6 \mathrm{fc}$, the darkest area. As can be seen from the analysis of variance table shown in Table 1 , the differences between these means cannot be attributed to chance. The results are also plotted in Figure 2. As shown in this figure, the expected relationship between brightness and pupil size is generally exhibited, although it is somewhat obscured by the large mean pupil size obtained for the brightest square $(18 \mathrm{fc})$. The correlation between brightness in footcandles and mean pupil size is indeed in the expected direction, although it is nonsignificant $(r=-.50)$.
For purposes of this study, however, the most important result is that the range of mean pupil sizes across all brightness levels was only $0.113 \mathrm{~mm}$, or $2.7 \%$ of the grand mean. The range between the brightest and darkest stimuli was only $0.009 \mathrm{~mm}$, or $0.2 \%$ of the grand mean $(4.119 \mathrm{~mm})$.

\section{DISCUSSION}

The change in pupil size in response to a wide range of intrastimulus brightness contrast variation was consistent with the results obtained by Hess (1975) and Woodmansee (Note 1), accounting for a range of $0.113 \mathrm{~mm}$, or $2.7 \%$. When a range of intrastimulus variation in illumination is employed, the obtained relationship may be far from perfect. In this present study, deviation from a strictly linear brightness/ pupil-size relationship, perhaps due to a position effect, resulted in the difference between the brightest and darkest square being negligible. However, upon closer inspection, it becomes clear that even the larger difference of $0.113 \mathrm{~mm}$ becomes negligible when compared to results of typical pupil research, which show pupil variation of much greater magnitude.

When it is considered that pupil responses to picture slide stimuli may average as high as from 0.3 to $0.8 \mathrm{~mm}$, a change of $10 \%$ to $20 \%$ (Hess, 1965 , 1972), then the $0.2 \%$ to $2.7 \%$ figures obtained in this study appear to be of limited significance in pupillometric experiments. Moreover, the intrastimulus brightness contrast of the experimental stimulus employed in this study is much greater than for most picture slide stimuli, especially if one follows Hess' (1972) instructions for minimizing such intrastimulus brightness contrast.

In pupillometric experiments, one should seek to minimize variation in intrastimulus brightness contrast, since this factor can affect pupil size. But its effects contribute, at best, only a small proportion of the variance of the pupil response to picture slide stimuli. Findings which have been obtained through the use of picture slide stimuli cannot be dismissed by the objection that there exist uncontrolled brightness variation within such stimuli, if such findings are of sufficiently large magnitude.

Table 1

One-Way ANOVA: Repeated Measures Across Subjects

\begin{tabular}{lrrrr}
\hline & df & $\begin{array}{r}\text { Sums of } \\
\text { Squares }\end{array}$ & F & p \\
\hline Source & 31 & 540.26 & & \\
Treatments & 4 & 308.06 & 3.70 & $<.01$ \\
Error & 124 & 2582.88 & & \\
\hline
\end{tabular}




\section{REFERENCE NOTE}

1. Woodmansse, J. J. An evaluation of pupil response as a measure of attitude toward Negroes. Progress Report, Attitude Research Project, Institute of Behavioral Science, University of Colorado, 1965.

\section{REFERENCES}

Goldwater, B. C. Psychological significance of pupillary movements. Psychological Bulletin, 1972, 77, 340-355.

Hess, E. H. Attitude and pupil size. Scientific American, 1965, 212, 46-54.
Hess, E. H. Pupillometrics: A method of studying mental, emotional and sensory processes. In N. S. Greenfield and R. A. Sternbach (Eds.), Handbook of psychophysiology. New York: Holt, Rinehart, and Winston, 1972. Pp. 491-531.

Hess, E. H. The tell-tale eye. New York: Van Nostrand Reinhold, 1975.

Jenisse, M. P. Pupillometry: Some advances, problems and solutions. In M. P. Janisse (Ed.), Pupillary dynamics and behavior. New York: Plenum Press, 1974. Pp. 1-8.

LoWenfeld, 1. Pupil size. Survey of ophthalmology, 1966, 11 , 291-294.

(Received for publication January 20, 1975; revision received April 28, 1975.) 